\title{
Polarization Effects on Thermally Stable Latency in Hollow- Core Photonic Bandgap Fibres
}

\author{
E. Numkam Fokoua ${ }^{1 *}$, W. Zhu ${ }^{1,2}$, Y. Chen ${ }^{1}$, S.R. Sandoghchi' ${ }^{1}$, T.D Bradley ${ }^{1}$, M.N. Petrovich ${ }^{1}$, D. J. \\ Richardson $^{1}$, F. Poletti ${ }^{1}$, and R. Slavík ${ }^{1}$ \\ 1. Optoelectronics Research Centre, University of Southampton, Southampton, SO17 1BJ, UK \\ 2. On leave from School of Optoelectronic Engineering and Instrumentation Science, Dalian University of Technology, Liaoning, \\ 116024, China. \\ *ernf1g10@orc.soton.ac.uk
}

\begin{abstract}
Hollow-Core fibers were recently demonstrated to have propagation time through them completely insensitive to changes in temperature. Here, we consider polarization dependent behaviour of these fibers and show how it can alter the fibre design. (C2019 Optical Society of America OCIS codes: (060.2320) Fiber optics amplifiers and oscillators, (140.3520) Laser, injection-locked.
\end{abstract}

\section{Introduction}

Optical fibres enable the propagation of optical signals over large distances. Although the intensity (power) of a propagating signal is relatively insensitive to changes in the ambient temperature, its phase $\phi$ and propagation time $\tau$ (latency) through the fibre are not [1]. This can pose significant challenges in applications requiring the dissemination of precise timing signals for synchronization purposes (e.g., FLASH in Desy, Hamburg), but increasingly in optical communications too, where the provision of stable and low latency is becoming a key requirement in many warehouse-scale datacenter applications or in $5 \mathrm{G}$ networks [2].

By guiding light in air rather than a glass material, hollow-core optical fibres provide the lowest possible latency for such applications $(>0.99 \mathrm{c})[3]$. Furthermore, we have recently shown that by carefully designing their structure, hollow-core photonic bandgap fiber can offer signal latencies completely insensitive to thermal changes [4]. In these proof-of-concept studies however, we utilized short fibre lengths and did not consider the effects of polarization or thermally-induced polarization changes.

In this work, we study for the first time (to the best of our knowledge) how the polarization properties of HC-PBGFs affect the thermal sensitivity of the latency in long fibre lengths. We show that the differential group delay (DGD) between orthogonal polarizations of the fundamental mode may in practice deteriorate the thermal stability of the latency when temperature changes induce polarization mode-coupling. To mitigate these adverse effects, improved fiber designs offering simultaneous low DGD and low delay thermal sensitivity are proposed for applications requiring low and thermally stable latency.

\section{Polarization effects in long HCPBGFs}

To study the impact polarization states might have on the thermal stability of latency in HC-PBGFs, we experimentally measured the thermal response of a 1-km long, 19c HC-PBGF sample (core diameter $31.5 \mathrm{um}$, transmission and cutback loss shown in Fig 1(b)) using the setup shown in Fig 1(a). A tunable laser source (TLS) was amplitude modulated with 7-GHz sine wave using an amplitude Mach-Zehnder modulator (MZM) and launched into the fibre under test (placed in a temperature controlled chamber) with the help of a polarization controller. The phase/time of arrival of the 7-GHz signal recovered at the fibre output was compared to the input one using an oscilloscope and the DGD was measured as min-max difference in signal arrival time when varying the input signal polarization. We repeated this DGD measurement across the spectral range covered by the TLS and for several temperatures of the fibre sample. DGD values recorded at room temperature $\left(30^{\circ} \mathrm{C}\right)$ and at $58^{\circ} \mathrm{C}$ are shown in Fig 1(b). The expected random nature of the DGD and thus dispersion derived from the data as a function of both wavelength and temperature can be clearly appreciated from Fig 1(c) and (d). Near $1610 \mathrm{~nm}$ (where measurements on a few meters of fibre showed thermally insensitive latency), the proximity of surface and cladding modes create large differential properties between the orthogonal polarizations of the fundamental mode, and the random DGD can be as high as $35 \mathrm{ps}$ or as low as $5 \mathrm{ps}$. This implies that under temperature changes, the propagation delay at this wavelength can change by as much as 30 ps purely due to the PMD/DGD, which is a value similar to delay of signal in a standard single mode fibers when experiencing change of ambient temperature of $1 \mathrm{~K}$ (as their thermal sensitivity is $40 \mathrm{ps} / \mathrm{km} / \mathrm{K}$ ). This represents noticeable deterioration of the performance. Away from the edges of the photonic bandgap or surface modes, the measured DGD and dispersion are much lower and more robust to thermal changes, suggesting that fibre designs targeting a zero delay thermal sensitivity closer to the centre of the bandgap should give the ultimate performance. 

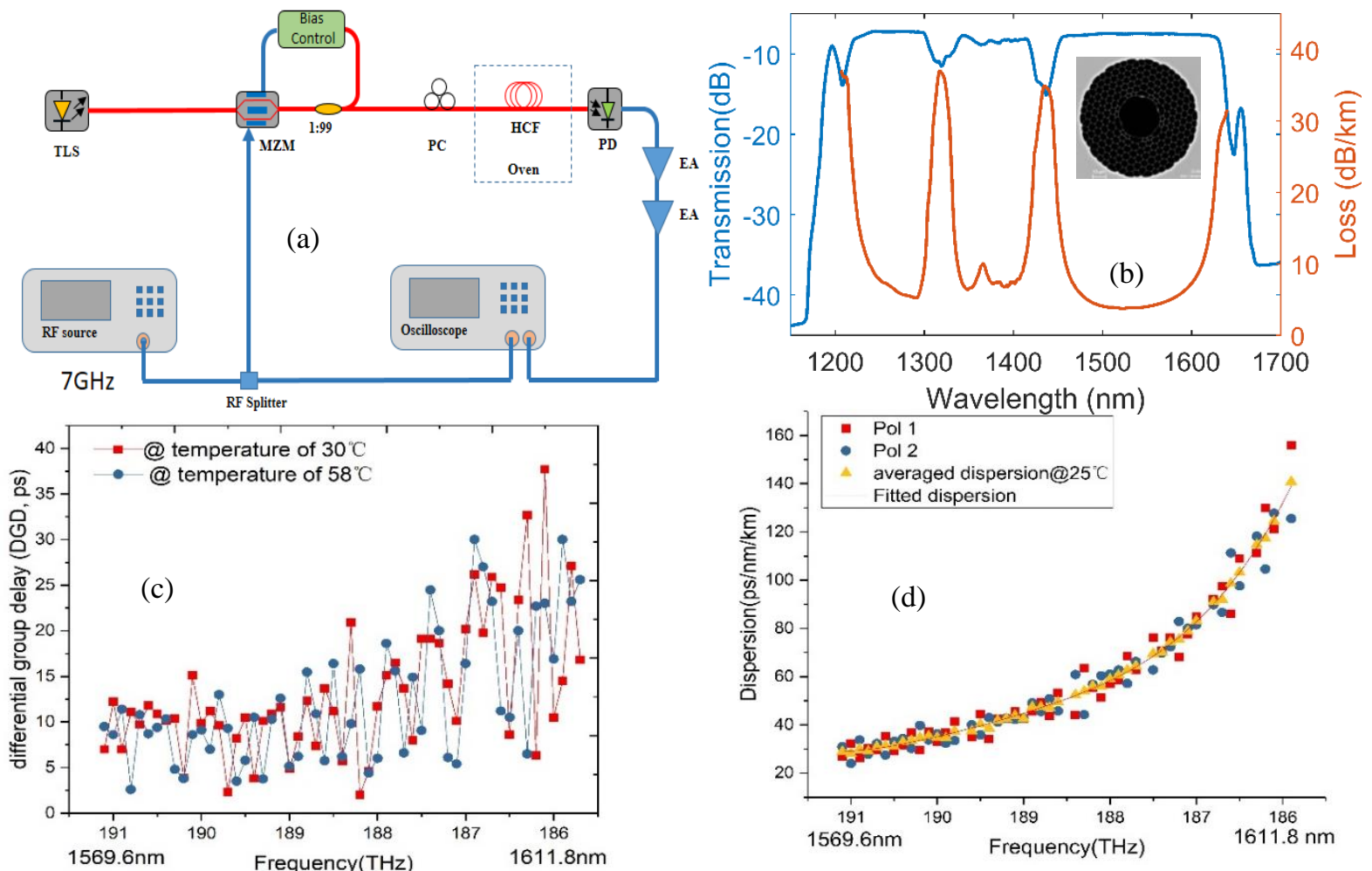

Fig.1: Experimental measurements of DGD and dispersion in a $1 \mathrm{~km}$ long 19c HC-PBGF sample at various temperatures. (a) Experimental setup (b) Measured short length transmission and cutback loss (4dB/km at $1550 \mathrm{~nm}$ ) (c) Measured DGD at 30 and $58 \mathrm{C}$ respectively $(\mathrm{d})$ measured dispersion at $25 \mathrm{C}$.

\section{Modelling and improved designs}

To verify this hypothesis, we first performed finite element simulations on the fabricated fiber sample we had measured. We obtained a permittivity profile of the fibre cross-section by reconstructing it from a high-resolution scanning electron micrograph (SEM) of the fiber end-face [5]. For such large core fibres, the interplay between viscous forces, surface tension and pressure differentials during the fabrication process typically results in residual core ellipticity and uneven thickness of the thin glass struts at the core boundary. These features introduce surface modes (see Fig 1b and 2a), which we expect to play the crucial role in the fiber's polarization properties [6]. The reconstructed profile was input into the commercial finite-element solver COMSOL Multiphysics and allowed us to simulate the fibre's key properties as summarized in Fig. 2. The simulated photonic bandgap extends from 1200 to $1670 \mathrm{~nm}$ with surface mode groups near $1320 \mathrm{~nm}, 1440 \mathrm{~nm}$, and $1650 \mathrm{~nm}$, in excellent agreement with the measured transmission of the fibre.

As discussed in our previous work [4], the thermal sensitivity of the latency in HC-PBGFs is dictated by the small but commensurate thermally-induced change in the material properties of the silica glass (e.g thermal expansion, thermo-optic and photoelastic effects). In addition to a small elongation, these effects cause a small shift of the photonic bandgap $(23 \mathrm{pm} / \mathrm{K})$ and impact the polarization-dependent fraction of light propagating in the glass. The overall thermal sensitivity of the fiber is quantified through the thermal coefficient of delay (TCD) [4]. Focusing our analysis on the widest useable transmission window of $1450-1650 \mathrm{~nm}$, we see from Fig 2 (b) that both polarizations of the fundamental mode have $\mathrm{TCD}=0$ near $1610 \mathrm{~nm}$. However, at this wavelength, they have a moderately high birefringence $(20 \mathrm{~cm}$ beat length), a large difference between their respective dispersion $(\Delta D=$ $82 \mathrm{ps} / \mathrm{nm} \cdot \mathrm{km}$ ) and their differential group delay is as high as $1.8 \mathrm{ps} / \mathrm{m}$. These results imply that for short lengths of fiber (e.g., few meters - as in our previous studies $[4,7]$ ) where negligible thermally-induced polarization modecoupling occurs, experimental measurements show polarization-independent thermally-stable latency. Over longer fibre lengths however, small changes in the fibre's structure along its length are more likely to occur, especially when subject to thermal fluctuations, and cause non-negligible polarization mode coupling. We estimate that with the calculated DGD value above, random mode-coupling after every $10 \mathrm{~m}$ can lead to an additional delay as long as 33 ps over a $1 \mathrm{~km}$ fibre length at $1610 \mathrm{~nm}$, again in good agreement with our experimental measurements. 

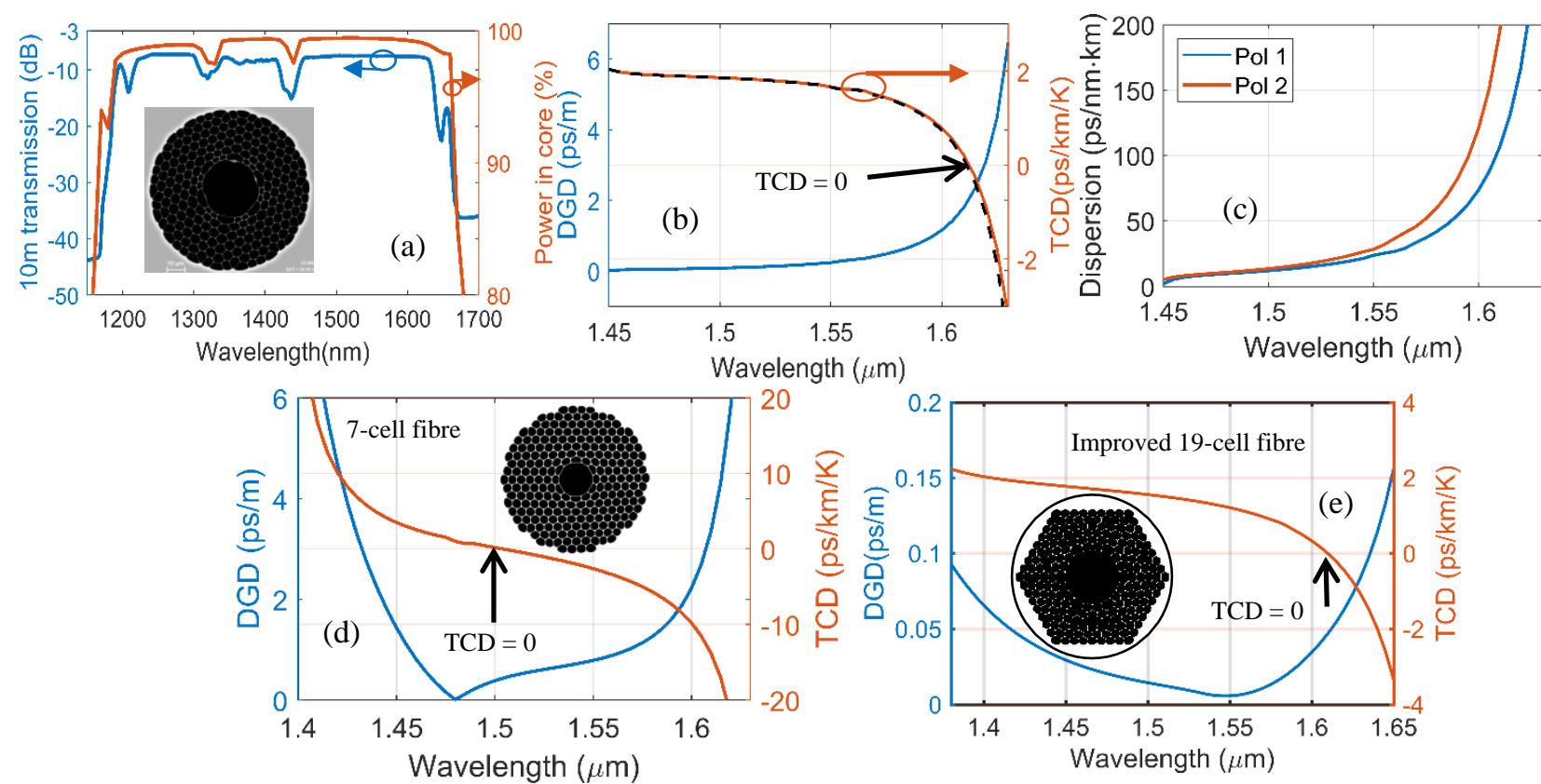

Figure 2: Modelling of polarization effects in a fabricated 19c HC-PBGFs. (a) Measured transmission over $10 \mathrm{~m}$ of fibre vs simulated power in the core. Inset is the SEM of the fibre end face. (b) DGD and TCD for both polarizations of the fundamental mode for the modelled cross-section (c) dispersion parameter for both polarizations of the fundamental mode as a function of wavelength. (d) DGD and TCD in a 7c fibre and (e) DGD and TCD in an improved 19c fibre design.

We conclude therefore that for ultrastable latency (>200x better than SMFs), in addition to targeting TCD $=0$, careful fibre design must also aim to minimize the DGD between polarization modes so that if present, thermally-induced polarization mode coupling doesn't impair the stability of the latency. Apart from preserving the fibre's symmetry as much as possible, a natural route to achieving this goal is to design the fibre so that its zero-TCD point is close to the middle of the photonic bandgap where differential properties between the polarization modes are the smallest, for example by using a HC-PBGFs with a smaller core-size. In Fig. 2(d), simulations results for a fabricated 7-cell fibre indicate indeed zero TCD near $1.5 \mathrm{um}$, where the DGD is only $0.2 \mathrm{ps} / \mathrm{m}$, nearly $10 \mathrm{x}$ lower than in the $19 \mathrm{c}$ fibre. Our initial modelling suggests that further reduction of the DGD between polarization modes is possible if we modify the design of the core surround by increasing the size of the glass nodes. Using this approach for a 19-cell fibre similar to the fabricated one we measured gives zero TCD at $1610 \mathrm{~nm}$, but with DGD as low as $0.04 \mathrm{ps} / \mathrm{m}$ (Fig 1(e)), paving the way for a fibre providing low, polarization-independent and ultrastable latency.

\section{Conclusions}

We have for the first time analysed both theoretically and experimentally the impact of polarization effects on the thermal stability of the low latency provided by long lengths of HC-PBGFs. We found that 7-cell fiber designs are less sensitive to these effects and further showed that in lower-loss 19-cell fiber design, the polarization effects at the zero thermal sensitivity point can be significantly reduced through fibre design (e.g., by a factor of 50 in our preliminary study), leading to significantly improved performance. This work is a crucial step towards fully evaluating and understanding the design trade-offs for HC-PBGFs links providing ultimately low and thermally stable latency.

This research has received funding from EPSRC under grand agreement EP/P030181/1 Airguide Photonics.

\section{References}

[1] V. Dangui et al, "Phase sensitivity to temperature of the fundamental mode in air-guiding photonic-bandgap fibers," Opt. Express 13,6669 (2005)

[2] H. Ballani et al, "Bridging the Last Mile for Optical Switching in Data Centers," Proc OFC 2018

[3] F. Poletti et al, "Towards high-capacity fibre-optic communications at the speed of light in vacuum," Nat. Photonics 7, 279-284 (2013)

[4] E. Numkam Fokoua et al "How to make the propagation time through an optical fiber fully insensitive to temperature variations," Optica 4, 659-668 (2017).

[5] E. Numkam Fokoua et al, "Accurate modelling of fabricated hollow-core photonic bandgap fibers," Opt. Express 23, 23117-23132 (2015)

[6] F. Poletti et al, "The effect of core asymmetries on the polarization properties of hollow core photonic bandgap fibers," Opt. Express 13, 9115-9124 (2005)

[7] R. Slavík et al, "Ultralow thermal sensitivity of phase and propagation delay in hollow core optical fibres, “ Sci. Rep. 5, 15447 (2015). 